\title{
The miRNA-184 drives renal fibrosis by targeting HIF1AN in vitro and in vivo
}

\section{Bin Chen ${ }^{1}$}

Received: 18 December 2017 / Accepted: 3 November 2018 / Published online: 10 December 2018

(c) The Author(s) 2018

\begin{abstract}
Progressive renal fibrosis is the last phase of chronic kidney disease and results in renal failure. Micro-RNA has been demonstrated as important agent to drive organ fibrosis. However, the precise mechanisms are not fully understood. Here, we found miRNA-184 as a critical mediator to promote the renal fibrosis by targeting HIF1AN. In Vivo, miRNA-184 expression levels remarkably increased both in patients' serum and in unilateral ureteral obstruction kidneys, as well as induced the expression of COL1A1 and COL3A1. Furthermore, transfection of NRK49F cells with miRNA-184 mimics down-regulated HIF1AN, transfection of NRK49F cells with miRNA-184 inhibitor up-regulated HIF1AN, while the cells transfected with miRNA-184 inhibitor exerted the opposite effect. When the cells were co-transfected with miRNA-184 mimics and HIF1AN, the expression of $\alpha$-SMA, GTGF, COL1A1, and COL3A1 at mRNA level was apparently decreased when compared with miRNA-184 mimic-transfected cells, which was strengthened when transfected with miRNA-184 inhibitor. Thus, miRNA-184 is an important agent to promote the fibrosis though binding to HIF1AN, and may be a promising novel target in treatment of renal fibrosis.
\end{abstract}

Keywords MiRNA-184 $\cdot$ HIF1AN $\cdot$ Renal fibrosis

\section{Introduction}

Renal fibrosis is the final stage that manifesting the chronic kidney disease and leading to impairment of kidney function $[1,2]$. A typical hallmark of tubule interstitial fibrosis is composed of myofibroblast accumulation, excessive deposition of extracellular matrix (ECM) as well as the renal tubules destruction [3, 4]. Other critical characteristics include accumulation of inflammatory leucocytes, which causes loss of renal function further [5, 6]. Fibrosis is key element of the high morbidity and mortality rates, especially related to some diseases, such as diabetic nephropathy. However, concrete therapeutic options for this target are not yet available in clinical application.

Recent studies show that miRNA can be involved in regulating numerous molecular and cellular processes, as well as its role in diseases and therapeutic potential [7-10].

Bin Chen

chenbinCBin@126.com

1 Kidney Department, Zhenhai People's Hospital of Ningbo City (Ningbo No.7 Hospital), 718 Nanerxi Road, Luotuo Subdistrict, Zhenhai, Ningbo, People's Republic of China
Micro-RNAs are a class of short noncoding RNAs of approximate 22 nucleotides in length, and they can regulate gene expression via post-translation and induction of mRNA degradation [11]. A certain number of miRNA have been considered to be associated with fibrotic processes in some relevant diseases, including miR-29, miR-192, and miR-21 [12-15]. It's reported that these miRNAs are induced by TGF- $\beta 1$ in renal cells [16], but another study demonstrates that TGF- 31 deduces miR-192 expression in human tubular epithelial cells [17]. The normalization of their expression could alleviate fibrosis in vitro and in vivo model, which suggests that focusing on these miRNAs could be a method to improve renal fibrosis disease [16].

Therefore, our study aimed to investigate miRNA profiling in the kidneys, and also to identify the potential mechanism in renal fibrosis. Moreover, we carried out intervention studies to determine the role of miRNA-184 in promoting the renal fibrosis. Finally, we determined whether suppressing the H1F1 might be a target by which miRNA-184 regulates renal impairment. 


\section{Materials and methods}

\section{Experimental animals}

A UUO kidney disease model was induced in C57 mice by left ureteral ligation in accordance with a previously described method [18]. After raising for 1 week, they were randomly divided into four group, one was sham surgery group with $10 \%$ DMSO intraperitoneal injection as control, and others were UUO models with CORM at doses of $2.5 \mathrm{mg} / \mathrm{kg}, 10 \mathrm{mg} / \mathrm{kg}$, and $15 \mathrm{mg} / \mathrm{kg}$, respectively, each group of 6 mice. Mice were killed after administration for 14 days, and the supernatant from eyeball blood was collected though centrifugation and stored at $-80^{\circ}$. Kidney tissues were collected and processed for evaluation of miR-184 as well as for immunohistochemistry, western blot, and real time PCR.

\section{Cell culture and transfection}

NRK49F cells were cultured in Dulbecco's modified Eagle's medium, $10 \%$ fetal calf serum (FCS), $1 \%$ glutamine, $1 \%$ nonessential amino acids, and antibiotics at $37{ }^{\circ} \mathrm{C}, 5 \%$ $\mathrm{CO}_{2}$ under humidified conditions. For the first time, we used Angiotensin II (R\&D Systems) to stimulate the cells to determine miRNA-184 expression levels. Moreover, NRK49F cells were respectively transfected with $30 \mathrm{nM}$ miRNA-184 mimics (Ambion, Austin, TX), miRNA-184 inhibitor (Ambion, Austin, TX), negative control miRNA mimics (Ambion), or miRNA-184 inhibitor in six well plates using siPort Neo-FX (Ambion) according to the manufacturer's instructions. After transient transfection, the cells were synchronized by culturing them in low-glucose medium without serum for $24 \mathrm{~h}$. NRK49F cells were then stimulated with AngII in accordance with our protocols.

\section{Quantitative RT-PCR}

The assay was carried out as previously described [19]. Quantitative RT (qRT)-PCR analyses of miR-184, HIF1AN mRNA, $\alpha$-SMA mRNA, GTGF mRNA, COL1A1 mRNA, and COL3A1 mRNA were carried out in RNA isolated from patients' serum or mice kidney tissue using specific TaqMan assays (Life Technologies). GAPDH was used as an internal control.

\section{Luciferase reporter assays}

NRK49F cells were co-transfected with a construct containing the human HIF1AN 3'untranslated region (UTR) downstream of the Firefly luciferase gene, the co-reporter vector PMIR-REPORT encoding the Renilla luciferase and
miR-184 mimics. After culturing for $36 \mathrm{~h}$, the activity of biluciferase was analyzed after cells were lysed with the passive lysis buffer by the Dual-Luciferase Reporter Assay System (Promega, Madison, WI, USA).

\section{Western blot}

Transfected NRK49F cells were also collected according to our protocols. Western blot analysis was carried out as previously described [20,21], with primary antibodies against $\alpha$-SMA (Sigma, US) and HIF1AN (Sigma, US). GAPDH was used as an internal control.

\section{Histology}

Changes in renal morphology were examined in H\&E and MASSON staining. The kidney tissues were fixed, fully automatic dehydrated, paraffin-embedded, and sliced into tissue sections $(4 \mathrm{~mm})$. All the slices were stained with hematoxylin for nuclei, and Masson's trichrome stain (Solarbio, China) was performed according to the manufacturer's specific instructions.

\section{Results}

\section{The miRNA-184 expression levels in vivo}

To determine whether miRNA-184 plays a role in the progression of kidney fibrosis, we detected the miRNA-184 expression levels in serum from both health group and observation group with kidney fibrosis, and to analyze the differences between the two groups. The results showed that miRNA-184 expression levels were significantly increased compared with the health group as shown in Fig. 1a. Meanwhile, we used the qRT-PCR to detect the levels of miRNA184 in mice kidney of sham operation group and of unilateral ureteral obstruction (UUO) group. Of note, the miRNA-184 expression levels in UUO group were significantly increased than those in the sham operation group as shown in Fig. 1b. These data indicated that miRNA-184 may play a key role in the development of fibrotic responses in the kidney.

\section{MiRNA-184 induces a-SMA expression in vitro}

We used NRK49F, a rat renal fibroblasts line, for mechanistic study to causal model of miRNA-184 on renal fibrosis. Angiotensin II, albuminuria and TGF- $\beta$ could contribute to tubule interstitial impairment and fibrosis in renal diseases [22, 23]. In order to investigate whether the stimuli could trigger miR-184 expression in renal fibroblasts line, we exposed NRK49F cells to AngII for $36 \mathrm{~h}$. Quantity RTPCR results showed that miRNA expression levels were 

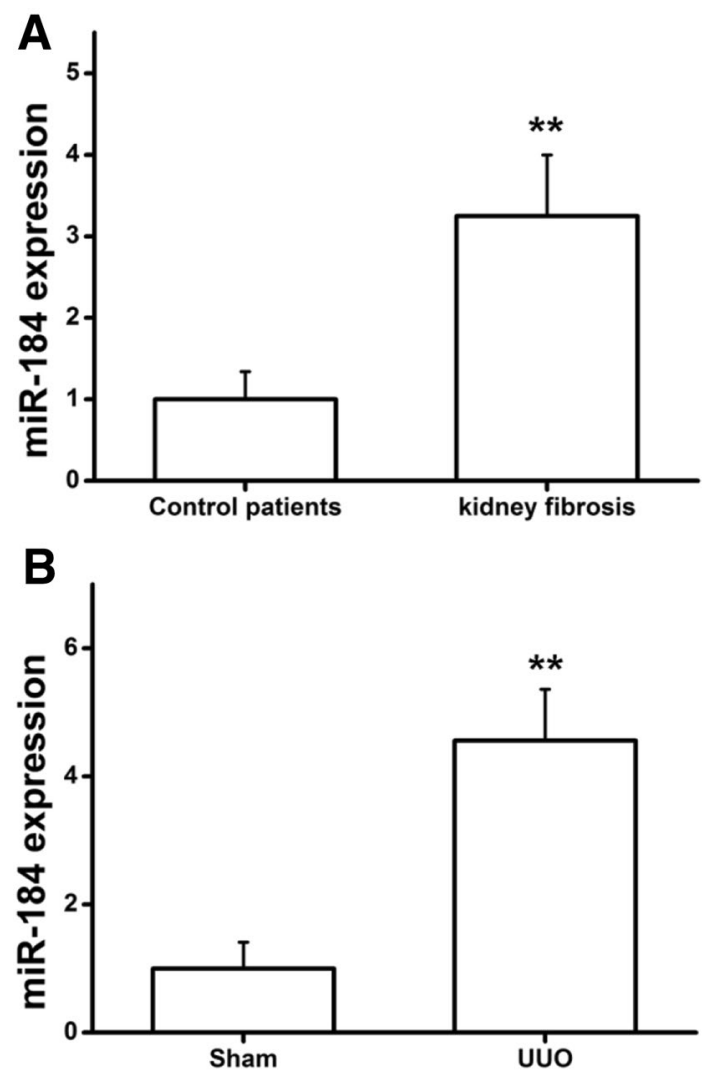

Fig. 1 MiRNA-184 expression levels in vivo. a miRNA-184 expression levels in both observation group and health group were determined by qRT-PCR assays. b miRNA-184 expression levels in mice kidney of sham operation group and of UUO group were determined by qRT-PCR assays. ${ }^{* *}$ Statistically highly significant, $P<0.01$

apparently increased after NRK49F was stimulated with AngII, as shown in Fig. 2a. Transfection of NRK49F cells with miRNA-184 mimics and under stimulation with AngII resulted in a significant increase in $\alpha$-SMA compared with negative control mimic-transfected cells. Western blotting analysis indicated that miRNA-184 could cause a sustained up-regulation of the $\alpha$-SMA expression (Fig. 2b).

\section{HIF1AN is a target of miRNA-184}

To demonstrate the target gene of miRNA-184, miRNA target prediction algorithms miRanda and EIMMo were employed together with the micro-RNA body map web tool. We focused on the HIF1AN (Fig. 3a) because we investigated the micro-RNA target gene database and made such a prediction. In order to prove that HIF1AN is a true miRNA184 target, we carried out luciferase reporter assay employing a plasmid pMIR-REPORT with full-length 3'-UTR of HIF1AN downstream of the luciferase gene. When the plasmid named pMIR-HIF1AN-wt was built, we performed overlapping PCR assay to construct plasmid pMIR-HIF1AN-mut

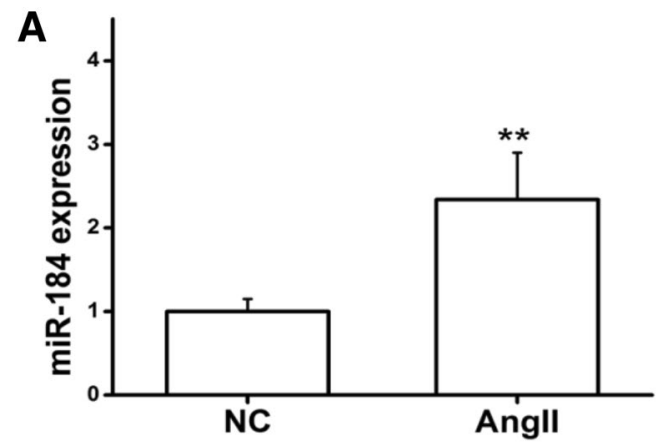

B

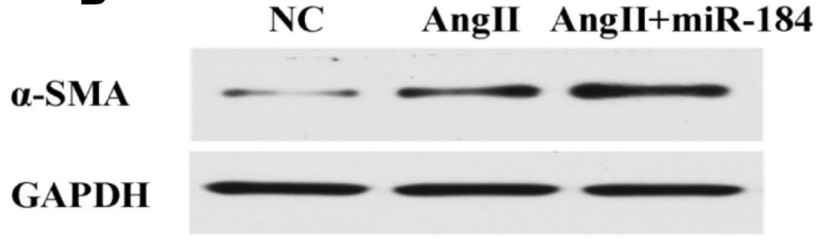

Fig. 2 MiRNA-184 induces $\alpha$-SMA expression in vitro. a miRNA184 expression levels stimulated with AngII were determined by qRT-PCR assays. b $\alpha$-SMA expression levels stimulated with AngII and AngII + miRNA-184 were determined by Western blotting analysis. **Statistically highly significant, $P<0.01$

\section{A

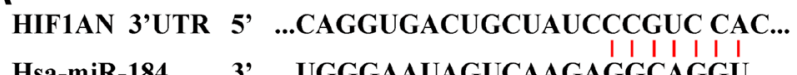 B PMIR-HIF1AN-WT ...CUAUCCCGUCCAC... pMIR-HIF1AN-Mut ...CUAUCGGCAGGUC...}

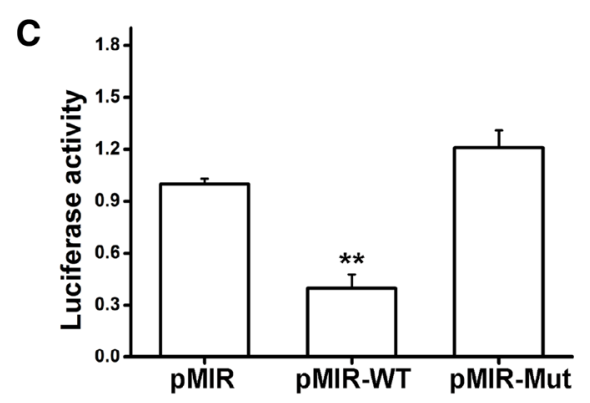

Fig. 3 HIF1AN is a target of miRNA-184. a 3'-UTR of HIF1AN binding to miRNA-184 as indicated. b The plasmid pMIR-HIF1ANmut was constructed by overlapping PCR assay. c Luciferase activity in NRK49F cells co-transfected with the reporter plasmid containing the HIF1AN 3'-UTR downstream of the Firefly luciferase gene, the co-reporter vector pMIR-REPORT encoding the Renilla luciferase and with miR-184 mimic. **Statistically highly significant, $P<0.01$

(Fig. 3b). To detect whether miRNA-184 could regulate the HIF1AN expression, NRF49F cells were co-transfected with miRNA-184 mimics and reporter plasmid. As shown 
in Fig. 3c, compared with pMIR-REPORT cells, it was showed that luciferase activity of pMIR-HIF1AN-mut was significant decreased, but that of pMIR-HIF1AN-mut had no statistical changes. Taken together, these results suggested that miRNA-184 could suppress HIF1AN expression by binding 3'-UTR of HIF1AN.

\section{MiRNA-184 regulates HIF1AN expression in mRNA and protein levels}

To estimate whether miRNA-184 could regulate HIF1AN in mRNA levels, we performed qRT-PCR assay to determine the mRNA levels of HIF1AN. As shown in Fig. 4a, transfection of NRF49F cells with miRNA-184 mimics showed a significant reduction in HIF1AN expression at mRNA levels compared with negative control miRNA mimic-transfected cells. As shown in Fig. 5a, transfection of NRF49F cells with miRNA-184 inhibitor showed a significant increase in HIF1AN mRNA compared with negative control miRNA mimic-transfected cells. Meanwhile, we carried out Western blot assay to detect the expression levels of HIF1AN. As shown in Fig. 4b, consistent with HIF1AN mRNA down-regulation, HIF1AN protein expression of NRF49F cells transfected with miRNA-184 mimics resulted in a significant reduction compared with negative control miRNA

A

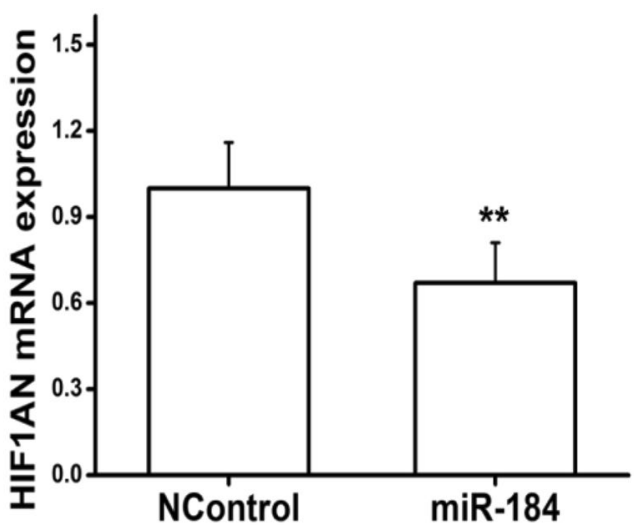

B

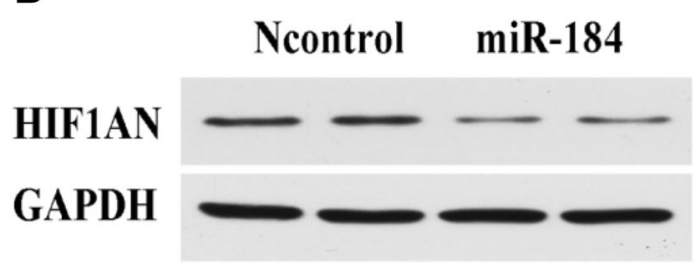

Fig. 4 MiRNA-184 regulates HIF1AN expression in mRNA and protein levels. Co-transfection of NRF49F cells with miRNA-184 mimics and negative control miRNA mimic. a HIF1AN mRNA expression was suppressed by miRNA-184. b HIF1AN protein expression was suppressed by miRNA-184.**Statistically highly significant, $P<0.01$ mimic-transfected cells. As shown in Fig. 5b, HIF1AN protein expression of NRF49F cells transfected with miRNA184 inhibitor resulted in a significant increase compared with negative control miRNA mimic-transfected cells. Based on the above results, we found that miRNA-184 could downregulate HIF1AN expression in mRNA and protein levels.

\section{MiRNA-184 promotes the progression of renal fibrosis inhibited by HIF1AN}

In order to validate that miRNA-184 promoted the progression of renal fibrosis by inhibiting HIF1AN expression, qRT-PCR assay was performed to determine the expression of $\alpha$-SMA, GTGF, COL1A1, and COL3A1. We identified that above indictors in cells transfected with miRNA-184 mimics were significantly increased compared with negative control miRNA mimic-transfected cells. However, $\alpha$-SMA, GTGF, COL1A1, and COL3A1 mRNA expression in cells co-transfected with miRNA-184 mimics and HIF1AN were apparently decreased when compared with miRNA-184 mimics-transfected cells (Fig. 6a-d). In addition, the expression of above parameters were significant down-regulated when transfected with miRNA-184 inhibitor (Fig. 7a-d). Notably, miRNA-184 promoted the progression of renal fibrosis, which could be inhibited by HIF1AN. In a word, HIF1AN involves in the progression of renal fibrosis mediated by miRNA- 184 .

\section{Renal fibrosis morphology in UUO mice associated with miRNA-184 modulation}

Based on the in vitro data indicating the effect of miRNA184 in the progression of renal fibrosis, we moved towards in vivo study to demonstrate the miRNA-184 regulated the consequent fibrosis. HE staining results showed that the structure of renal tubules and glomerulus were clear, the epithelial cells arranged neatly, the basement membrane of small organs was intact, and no obvious cell infiltration was observed in the interstitium in the sham-operated mice. However, in the UUO mice, there was renal glomerular dilatation, epithelial cell degeneration, and necrosis, indicating UUO kidney was successfully modeled. The injection of miRNA-184 into the tail vein resulted in more pronounced renal tubular dilatation and increased degeneration and necrosis of epithelial cells. Moreover, we analyzed the changes of renal fibrosis in mice performing Masson's trichrome staining. The results showed that the area of renal fibrosis and extracellular matrix deposition were significantly increased in UUO mice with miRNA-184 injection compared with sham-operated mice and UUO mice (Fig. 8a). Consistent with in vitro results of COL1A1 and COL3A1 mRNA expression, we found that the expression levels of COL1A1 and COL3A1 mRNA in sham-operated 
A

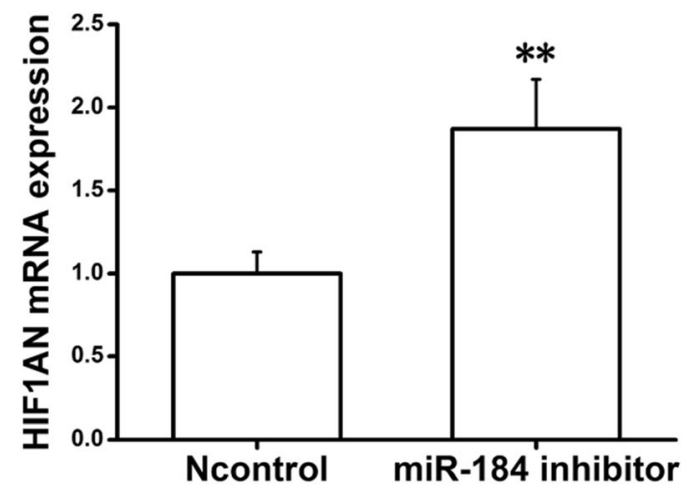

B

\section{HIF1AN}

\section{GAPDH}

\section{Ncontrol miR-184 inhibitor}

Fig. 5 MiRNA-184 inhibitor regulates HIF1AN expression in mRNA and protein levels. Co-transfection of NRF49F cells with miRNA184 inhibitor or negative control miRNA mimic. a HIF1AN mRNA expression was up-regulated by miRNA-184 inhibitor. b HIF1AN protein expression was up-regulated by miRNA-184 inhibitor. **Statistically highly significant, $P<0.01$ mice and UUO mice were significantly decreased than those in UUO mice injected with miRNA-184 (Fig. 8b, c). The above results showed that miRNA-184 could significantly promote progression of renal fibrosis.

\section{Statistical analysis}

Descriptive statistics were calculated and shown in figures. One-way ANOVA analysis was performed for multiple group comparisons. The Student's $t$-test was used for comparison between two groups. $P$ values less than 0.05 was considered statistically significant difference. The statistical analysis was performed using SPSS software (SPSS for windows 17.0, SPSS Inc.).

\section{Discussion}

Renal fibrosis is the final common stage of any form of progressive kidney disease, resulting in renal impairment. For a long-term study, we have been exploring the relevant molecular mechanisms involving in the development of renal fibrosis and identified that the miR-324-3p/Prep complex and miR-433 exerted effect during the fibrotic process [24, 25]. Here, we focused on miRNA-184 and demonstrated that is a key agent during the renal fibrosis.

Little information is available pertaining to miRNA-184 expression in the kidney. Some reporters showed that renal miRNA profiling of rodents displayed upregulated miR-184
Fig. 6 MiRNA-184 promotes the progression of renal fibrosis inhibited by HIF1AN. Cotransfection of NRF49F cells with miRNA-184 mimics, negative control miRNA mimic and miRNA-184 mimics + HIF1AN. a $\alpha$-SMA mRNA expression. b GTGF mRNA expression. c COL1A1 mRNA expression. $\mathbf{d}$ COL3A1 mRNA expression. **Statistically highly significant, $P<0.01$
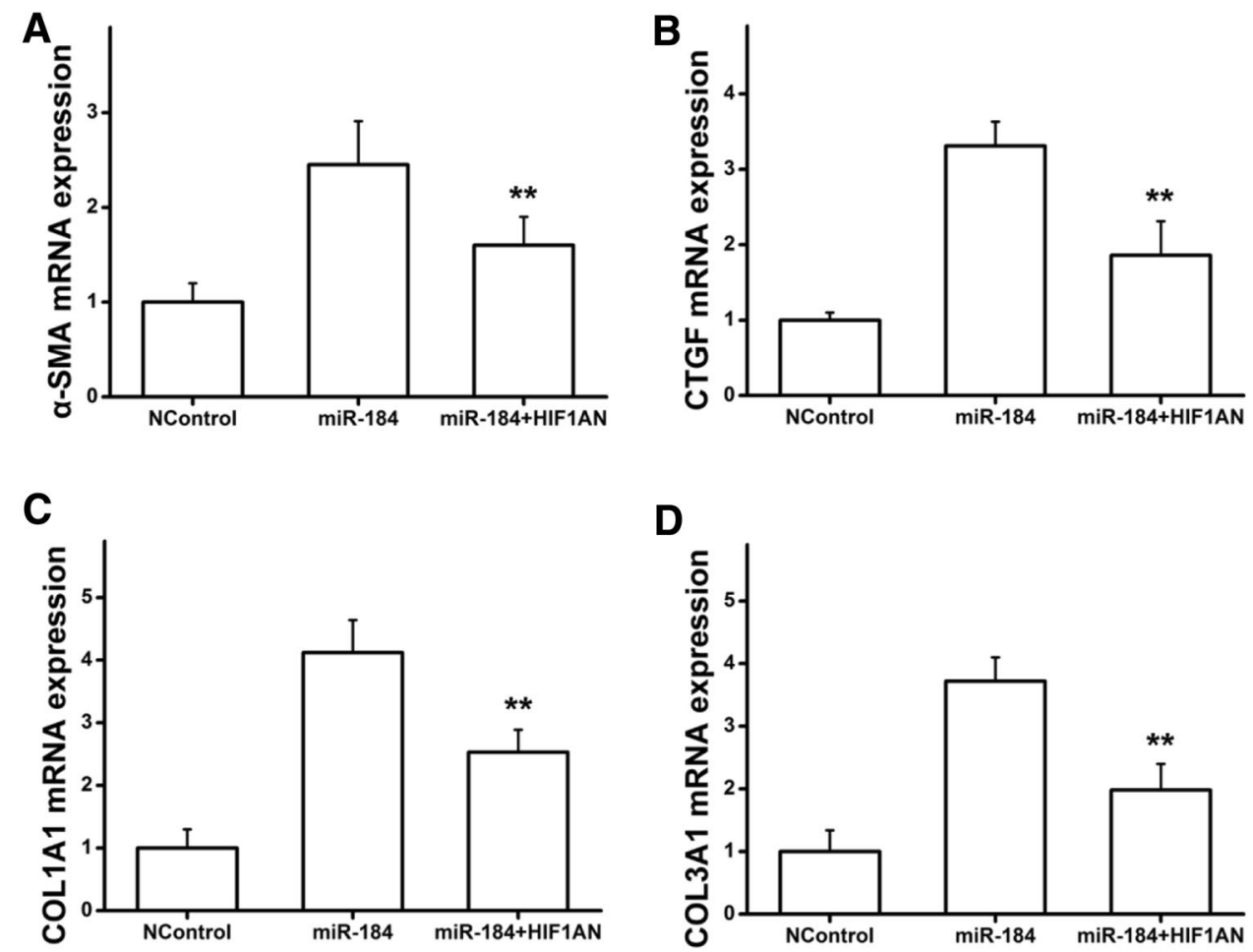

D

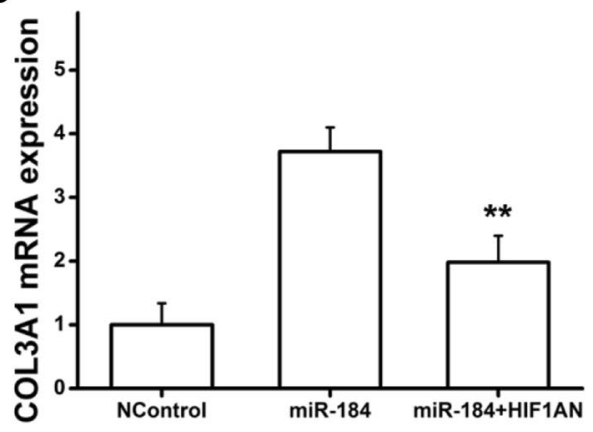



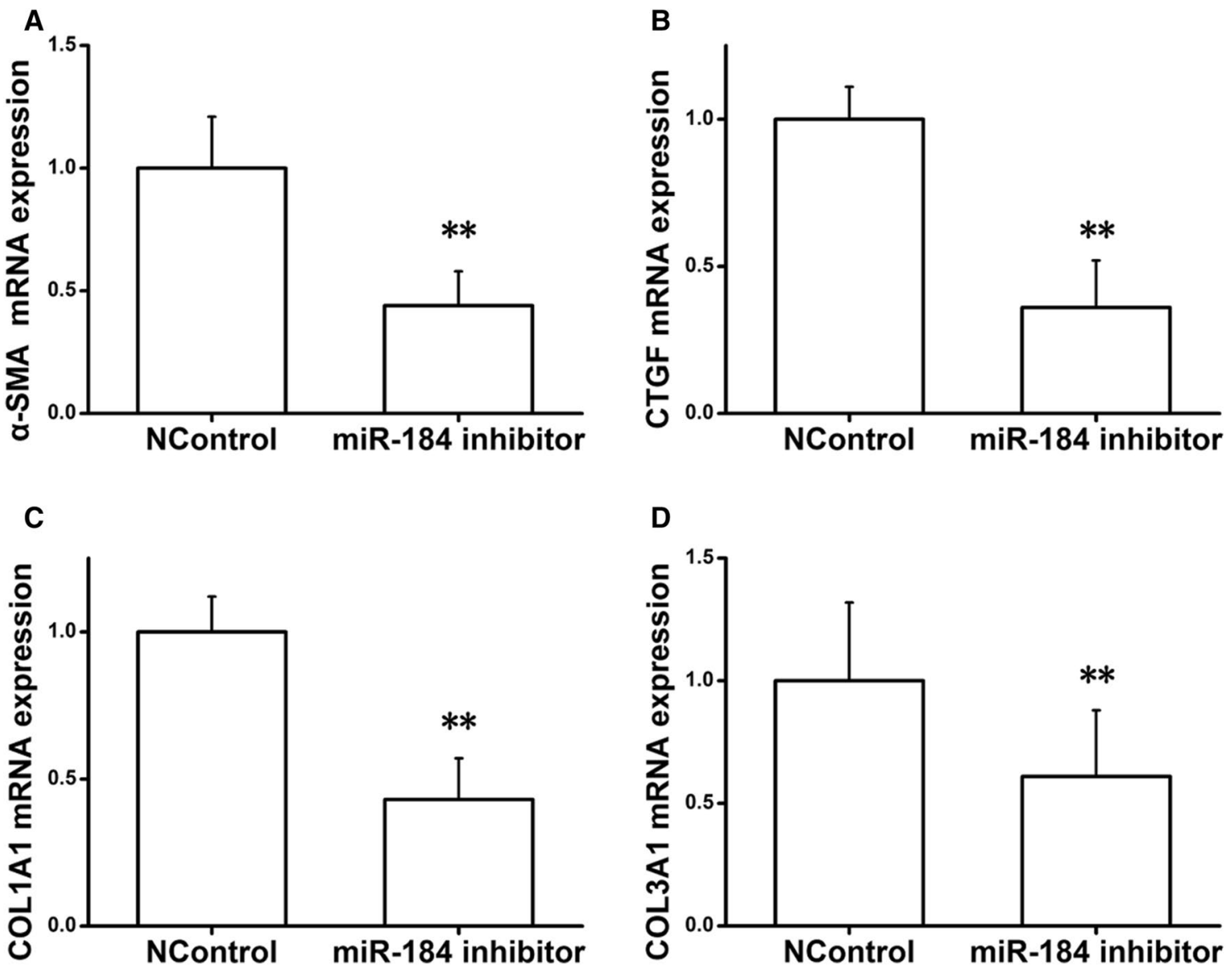

Fig. 7 The effect miRNA-184 inhibitor at miRNA expression levels of $\alpha$-SMA, GTGF, COL1A1 and COL3A1. Co-transfection of NRF49F cells with miRNA-184 inhibitor. a $\alpha$-SMA mRNA expres-

in aged kidneys, indicating that epigenetic regulation of renal aging likely occurred via inhibiting miRNA-184 targeted genes [26, 27]. Zan chi et al. [28] established a link between abnormal tubular miR-184 and tubule interstitial fibrosis in the diabetic kidneys through suppression of the target LPP3, which plays a critical role in modulating biosynthesis of lipid phosphates involved in multi-organ fibrosis and in cell signal transduction [29, 30]. In this study, we added new finding that miRNA-184 is a positive agent binding HIF1AN as a potential target during the fibrosis. HIF1AN, an asparaginyl (Asn) hydroxylase, is able to modulate the activities of important biological regulators (such as HIF1a [31], IкB [32], Notch [33]) through hydroxylation. It has been reported that HIF- $1 \alpha$ exerted an effect in hypoxia- and chronic kidney disease-induced fibrosis, while genetic knock-down of HIF-1 $\alpha$ in renal epithelial cells led to a significant reduction in collagen deposition, including COL1A1 and COL3A1 [34]. Mahon et al. revealed that HIF1AN considered as an important inhibitor can interact with Hypoxia-inducible factor (HIF)- $1 \alpha$ to negatively

sion. b GTGF mRNA expression. c COL1A1 mRNA expression. d COL3A1 mRNA expression. **Statistically highly significant, $P<0.01$

regulate the HIF- $1 \alpha$ transcriptional activity [35]. Therefore, HIF1AN can be regarded as a critical agent implicated in the process of renal fibrosis.

It's reported that Micro-RNAs suppress their target gene though binding their $3^{\prime}$ UTR to induce protein translational inhibition or mRNA degradation [31]. In order to identify whether miRNA-184 could target HIF1AN gene, we detected the expression levels of HIF1AN in rat kidney fibroblasts overexpressing miRNA-184 in the miRNA and protein levels. Notably, both mRNA and protein levels of HIF1AN were apparently inhibited by miRNA-184 when compared with the control (Fig. 4a, b). While both mRNA and protein levels of HIF1AN were apparently promoted by miRNA-184 inhibitor when compared with the control (Fig. 5a, b). To further determine whether miRNA-184 modulates HIF1AN by binding its $3^{\prime}$ UTR, we generated luciferase reporter linked to full-length $3^{\prime}$-UTR of HIF1AN and NRF49F cells were co-transfected with miRNA-184 mimics and reporter plasmid. Compared with pMIR-REPORT cells, it was showed that luciferase activity of pMIR-HIF1AN-wt 

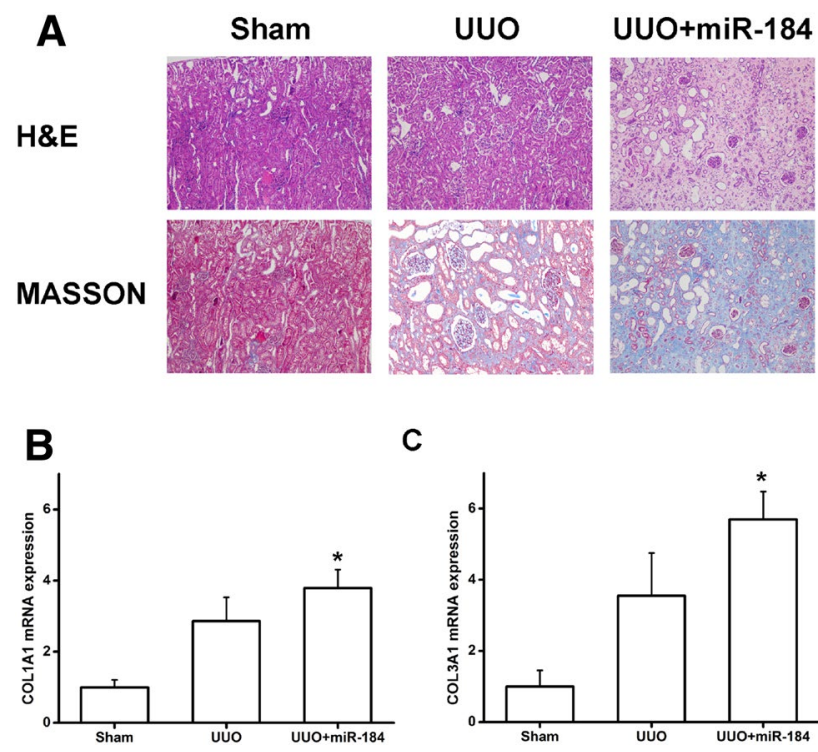

C

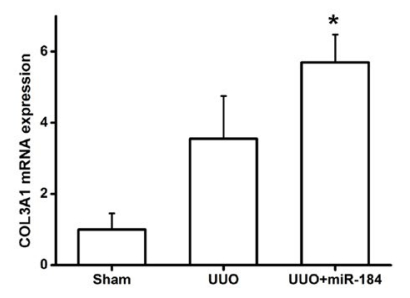

Fig. 8 Renal fibrosis morphology in UUO mice associated with miRNA-184 modulation. UUO mice model was established and make an injection of miRNA-184 into the tail vein. a HE and MASSON staining of mice kidney in various groups. b COL1A1 mRNA expression. c COL3A1 mRNA expression. *Statistically significant, $P<0.05$

was significantly decreased, which indicated that miRNA184 suppressed its target gene HIF1AN expression by specifically binding its 3'-UTR. Above findings of target gene of miRNA-184 were consistent with the theory that the target sites of micro-RNA could be enriched in multiple components of the same signaling pathway $[36,37]$.

Angotens in II is a critical mediator of proteinuria and fibrosis, which failed to induce miRNA-184 expression in rat kidney cells. It's able to express angiotensin II type 1 receptors [38], suggesting that angiotensin II is lack of a direct effect on miRNA-184. In contrast, one interesting finding of our study is that Angiotensin II is an effective trigger for miRNA-184 expression in rat kidney fibroblasts (Fig. 2a). In addition, Western blotting analysis revealed that miRNA184 could cause a sustained up-regulation of the $\alpha$-SMA, CTGF, COL1A1, and COL3A1 expression (Figs. 2b, 6a, b). And miRNA-184 inhibitor could cause a sustained downregulation of the $\alpha$-SMA, CTGF, COL1A1 and COL3A1 expression (Fig. 7a, b). The fibrotic markers, such as collagens [39-41], could be induced involved in the signaling conduction pathway to promote the fibrosis and could be control involved in the signaling conduction pathway to suppress the fibrosis. Previous studies [42-44] described that TGF $\beta 1$ enhanced $\alpha$-SMA, COL1A1, COL3A1, and CTGF levels to drive renal fibrosis mediated by the miR-433. In cardiac fibrosis, elevated COL3A1 has been described in early myocardial remodeling, while COL1A1 accumulation is observed at a later stage [45]. Importantly, in UUO model, we confirmed that the expression levels of COL1A1 and COL3A1 mRNA in mice injected with miRNA-184 were significantly elevated (Fig. 8b), indicating miRNA-184 may play a critically pathological role in fibrosis. In addition, we had a new finding that HIF1AN, a target of miRNA-184 during renal fibrosis, exerts its ability to regulate miRNA184-induced the expression of fibrotic markers to facilitate fibrosis.

In conclusion, the present study provides the novel finding that miRNA-184 plays a role in renal fibrosis by targeting HIF1AN. Combination of in vitro and in vivo data showed the opportunity that targeting miRNA-184 in association with angiotensin II lowing medicine to have potential effect in renal injury disease. However, whether miRNA-184 is a rooted factor or it has an additional role in the development of renal fibrosis needs to be further studied.

\section{Compliance with ethical standards}

Conflict of interest The authors declare no competing financial interests.

Ethical approval All applicable international, national, and/or institutional guidelines for the care and use of animals were followed. And all procedures performed in studies involving human participants were in accordance with the ethical standards of the institutional and/ or national research committee and with the 1964 Helsinki declaration and its later amendments or comparable ethical standards.

Open Access This article is distributed under the terms of the Creative Commons Attribution 4.0 International License (http://creativeco mmons.org/licenses/by/4.0/), which permits unrestricted use, distribution, and reproduction in any medium, provided you give appropriate credit to the original author(s) and the source, provide a link to the Creative Commons license, and indicate if changes were made.

\section{References}

1. Remuzzi G, Bertani T (1998) Pathophysiology of progressive nephropathies. N Engl J Med 339(20):1448-1456

2. Iwano M, Neilson EG (2004) Mechanisms of tubulointerstitial fibrosis. Curr Opin Nephrol Hypertens 13(3):279-284

3. Müller GA, Zeisberg M, Strutz F (2000) The importance of tubulointerstitial damage in progressive renal disease. Nephrol Dial Transpl 15(Suppl 6):76

4. Deng C, Zheng J, Wan W et al (2013) Suppression of cell proliferation and collagen production in cultured, human hypertrophic scar fibroblasts by Sp1 decoy oligodeoxynucleotide. Mol Med Rep 7(3):785

5. Navarro-González JF, Mora-Fernández C, Muros de Fuentes M, García-Pérez J (2011) Inflammatory molecules and pathways in the pathogenesis of diabetic nephropathy. Nat Rev Nephrol 7(6):327-340

6. Loeffler I, Wolf G (2015) Epithelial-to-mesenchymal transition in diabetic nephropathy: fact or fiction? Cells 4(4):631-652

7. Chung AC, Dong Y, Yang W,et al (2013) Smad7 suppresses renal fibrosis via altering expression of TGF- $\beta / \mathrm{Smad} 3$-regulated microRNAs. Mol Ther J Am Soc Gene Ther 21(2):388 
8. Lorenzen JM, Haller H, Thum T (2011) MicroRNAs as mediators and therapeutic targets in chronic kidney disease. Nat Rev Nephrol 7(5):286-294

9. Kato M, Natarajan R (2015) MicroRNAs in diabetic nephropathy: functions, biomarkers, and therapeutic targets. Ann NY Acad Sci 1353:72

10. Zhong X, Chung ACK, Chen HY et al (2013) miR-21 is a key therapeutic target for renal injury in a mouse model of type 2 diabetes. Diabetologia 56(3):663-674

11. Zavadil J, Narasimhan M, Blumenberg M et al (2007) Transforming growth factor-beta and microRNA:mRNA regulatory networks in epithelial plasticity. Cells Tissues Organs 185(1-3):157

12. Wang G, Kwan BC, Lai FM et al (2012) Urinary miR-21, miR29, and miR-93: novel biomarkers of fibrosis. Am J Nephrol 36(5):412

13. Kato M, Natarajan R (2014) Diabetic nephropathy-emerging epigenetic mechanisms. Nat Rev Nephrol 10(9):517-530

14. Kato M, Zhang J, Wang M et al (2007) MicroRNA-192 in diabetic kidney glomeruli and its function in TGF- $\beta$-induced collagen expression via inhibition of E-Box repressors. Proc Natl Acad Sci USA 104(9):3432

15. Qin W, Chung AC, Huang XR et al (2011) TGF- $\beta /$ Smad3 signaling promotes renal fibrosis by inhibiting miR-29. J Am Soc Nephrol 22(8): 1462

16. Zoja C, Cattaneo S, Fiordaliso F et al (2011) Distinct cardiac and renal effects of ETA receptor antagonist and ACE inhibitor in experimental type 2 diabetes. Am J Physiol Ren Physiol 301(5):F1114

17. Krupa A, Jenkins R, Luo DD et al (2010) Loss of MicroRNA-192 promotes fibrogenesis in diabetic nephropathy. J Am Soc Nephrol 21(3):438

18. Lan HY, Mu W, Tomita $\mathrm{N}$ et al (2003) Inhibition of renal fibrosis by gene transfer of inducible Smad7 using ultrasound-microbubble system in rat UUO model. J Am Soc Nephrol 14(6):1535

19. Liu G, Friggeri A, Yang Y et al (2009) miR-147, a microRNA that is induced upon Toll-like receptor stimulation, regulates murine macrophage inflammatory responses. Proc Natl Acad Sci 106(37):15819-15824

20. Yang F, Huang XR, Chung AC et al (2010) Essential role for Smad3 in angiotensin II-induced tubular epithelial-mesenchymal transition. J Pathol 221(4):390

21. Koka V, Xiao RH, Chung ACK et al (2008) Angiotensin II upregulates angiotensin I-converting enzyme (ACE), but down-regulates ACE2 via the AT1-ERK/p38 MAP kinase pathway. Am J Pathol 172(5):1174-1183

22. Arora MK, Singh UK (2013) Molecular mechanisms in the pathogenesis of diabetic nephropathy: an update. Vascul Pharmacol 58(4):259-271

23. Macconi D, Remuzzi G, Benigni A (2014) Key fibrogenic mediators: old players. Renin-angiotensin system. Kidney Int Suppl 4(1):58-64

24. Macconi D, Tomasoni S, Romagnani P et al (2012) MicroRNA324-3p promotes renal fibrosis and is a target of ACE inhibition. J Am Soc Nephrol 23(9):1496-1505

25. Li R, Chung AC, Dong K et al (2013) The microRNA miR-433 promotes renal fibrosis by amplifying the TGF- $\beta /$ Smad3-Azin 1 pathway. Kidney Int 84(6):1129-1144

26. Bai XY, Ma Y, Ding R et al (2011) miR-335 and miR-34a Promote renal senescence by suppressing mitochondrial antioxidativeenzymes. J Am Soc Nephrol 22(7):1252
27. Liu X, Fu B, Chen D et al (2015) miR-184 and miR-150 promote renal glomerular mesangial cell aging by targeting Rab1a and Rab31. Exp Cell Res 336(2):192-203

28. Zanchi C, Macconi D, Trionfini P et al (2017) MicroRNA-184 is a downstream effector of albuminuria driving renal fibrosis in rats with diabetic nephropathy. Diabetologia 60(6):1114-1125

29. Sciorra VA, Morris AJ (1999) Sequential actions of phospholipase $\mathrm{D}$ and phosphatidic acid phosphohydrolase $2 \mathrm{~b}$ generate diglyceride in mammalian cells. Mol Biol Cell 10(11):3863

30. Brindley DN, English D, Pilquil C et al (2002) Lipid phosphate phosphatases regulate signal transduction through glycerolipids and sphingolipids. Biochim Biophys Acta 1582(1-3):33

31. Lando D, Peet DJ, Gorman JJ et al Lando D, Peet DJ, Gorman JJ, Whelan DA, Whitelaw ML, Bruick RK (2002) FIH-1 is an asparaginyl hydroxylase enzyme that regulates the transcriptional activity of hypoxia-inducible factor. Genes Dev 16:1466-1471

32. Devries IL, Hampton-Smith RJ, Mulvihill MM et al (2010) Consequences of IkappaB alpha hydroxylation by the factor inhibiting HIF (FIH). Febs Lett 584(23):4725

33. Chan MC, Atasoylu O, Hodson E et al (2015) Potent and selective triazole-based inhibitors of the hypoxia-inducible factor prolyl-hydroxylases with activity in the murine brain. PLoS ONE 10(7): 0132004

34. Nayak BK, Shanmugasundaram K, Friedrichs WE et al (2016) HIF-1 mediates renal fibrosis in OVE26 type 1 diabetic mice. Diabetes 65(5): 1387

35. Mahon PC, Hirota K, Semenza GL (2001) FIH-1: a novel protein that interacts with HIF- $1 \alpha$ and VHL to mediate repression of HIF-1 transcriptional activity. Genes Dev 15(20):2675

36. Tsang JS, Ebert MS, Oudenaarden AV (2010) Genome-wide dissection of MicroRNA functions and cotargeting networks using gene set signatures. Mol Cell 38(1):140

37. Zhou L, Xue H, Yuan P et al (2010) Angiotensin AT1 receptor activation mediates high glucose-induced epithelial-mesenchymal transition in renal proximal tubular cells. Clin Exp Pharmacol Physiol 37(9):e152-e157

38. Chung AC, Lan HY (2011) Chemokines in renal injury. J Am Soc Nephrol 22:802-809

39. Li ZI, Chung AC, Zhou L et al (2011) C-reactive protein promotes acute renal inflammation and fibrosis in unilateral ureteral obstructive nephropathy in mice. Lab Investig 91(6):837-851

40. Liu F, Chen HY, Huang XR et al (2011) C-reactive protein promotes diabetic kidney disease in a mouse model of type 1 diabetes. Diabetologia 54(10):2713

41. Landau G, Bercovich Z, Park MH et al (2010) The role of polyamines in supporting growth of mammalian cells is mediated through their requirement for translation initiation and elongation. J Biol Chem 285(17):12474-12481

42. Cucoranu I, Clempus R, Dikalova A et al (2005) NAD(P)H oxidase 4 mediates transforming growth factor-beta1-induced differentiation of cardiac fibroblasts into myofibroblasts. Circ Res 97:900-907

43. Roberts AB (1998) Molecular and cell biology of TGF-beta. Miner Electrol Metab 24(2-3):111-119

44. Zhang R, Zhang YY, Huang XR et al (2010) C-reactive protein promotes cardiac fibrosis and inflammation in angiotensin IIinduced hypertensive cardiac disease. Hypertension 55(4):953

45. Graham HK, Horn M, Trafford AW (2008) Extracellular matrix profiles in the progression to heart failure. Acta Physiol 194(1):3-21 